\title{
ESTUDO DA DESIDRATAÇÃO OSMÓTICA DE TOMATE EM SOLUÇÕES TERNÁRIAS PELA METODOLOGIA DE SUPERFÍCIE DE RESPOSTA ${ }^{1}$
}

\author{
Renata Valeriano TONON², Alessandra Faria BARONI², Míriam Dupas HUBINGER ${ }^{2, *}$
}

\begin{abstract}
RESUMO
O objetivo deste trabalho foi estudar a influência da temperatura $\left(20-40{ }^{\circ} \mathrm{C}\right)$, da composição da solução $(0 \% \mathrm{NaCl} / 65 \%$ sacarose - $10 \% \mathrm{NaCl} / 55 \%$ sacarose) e do nível de agitação $(0-1000 \mathrm{rpm})$, na desidratação osmótica de metades de tomate. O processo foi realizado em um tanque encamisado conectado a um banho termostático, sendo a agitação da solução osmótica promovida por um agitador mecânico e medida por um tacômetro digital. Os resultados foram analisados por metodologia de superfície de resposta, de acordo com um planejamento experimental fatorial $2^{3}$ completo. A perda de água, o ganho de sal e de sacarose e a atividade de água final dos produtos foram influenciados positivamente pela temperatura e pelo aumento do teor de sal na solução. A agitação influenciou somente a perda de água, indicando que, neste caso, a transferência de massa não é governada apenas por um mecanismo interno de difusão. A proximidade do equilíbrio atingida em cada ensaio foi verificada pela relação entre as atividades de água da solução e das amostras. As amostras processadas em soluções com maior quantidade de sal foram as que se apresentaram mais distantes do equilíbrio, após $3 \mathrm{~h}$ de processo.

Palavras-chave: Lycopersicum esculentum, desidratação osmótica, transferência de massa, planejamento experimental, atividade
\end{abstract} de água.

\section{SUMMARY}

STUDY OF OSMOTIC DEHYDRATION OF TOMATO IN TERNARY SOLUTIONS THROUGH RESPONSE SURFACE METHODOLOGY. ThE objective of this work was to study the influence of temperature $\left(20-40{ }^{\circ} \mathrm{C}\right)$, solution composition $(0 \% \mathrm{NaCl} / 65 \% \mathrm{sucrose}-10 \% \mathrm{NaCl} / 55 \%$ sucrose) and agitation level $(0-1000 \mathrm{rpm})$, on the osmotic dehydration of tomato's halves. The process was carried out in a jacketed tank connected to a thermostatic bath, and osmotic solution agitation was promoted by a mechanical stirrer and measured by a digital tachometer. Results were analyzed through response surface methodology, according to a $2^{3}$ factorial experimental design. Water loss, salt and sucrose gain and final water activity of the product were positively influenced by temperature and by increasing salt content on the solution. Agitation influenced only on water loss, indicating that in this case, mass transfer is not governed only by a diffusion internal mechanism. Equilibrium proximity reached in each test was verified through the relationship between solution and samples water activities. The samples processed in solutions with higher salt content seemed to be more distant of equilibrium, after 3 hours of process.

Keywords: Lycopersicum esculentum, osmotic dehydration, mass transfer, experimental desing, water activity.

\section{1 - INTRODUÇÃO}

O tomate é um dos vegetais mais consumidos no mundo, tanto na forma in natura (em saladas), como na forma industrializada (em molhos, sucos, ketchup ou mesmo seco), sendo o segundo vegetal em área cultivada e o primeiro em volume industrializado. No Brasil, os maiores produtores são os Estados de Goiás, São Paulo, Minas Gerais, Pernambuco e Bahia, responsáveis por $77 \%$ da produção anual de tomate [1]. Em 2004, a produção nacional foi superior a 3.400 .000 toneladas, correspondente a aproximadamente $21 \%$ da produção total de hortaliças no País [9].

Nos últimos anos, o tomate vem sendo considerado um alimento funcional, graças ao alto teor de licopeno pre-

\footnotetext{
${ }^{1}$ Recebido para publicação em 10/10/2005. Aceito para publicação em 6/7/2006 (001691)

${ }^{2}$ Universidade Estadual de Campinas,

Faculdade de Engenharia de Alimentos,

Departamento de Engenharia de Alimentos,

C. P. 6121, CEP 13083-970, Campinas (SP), Brasil,

E-mail:mhub@fea.unicamp.br

${ }^{3}$ Escola de Engenharia Mauá,

Instituto Mauá de Tecnologia,

Praça Mauá 1, CEP 09580-900, São Caetano do Sul (SP), Brasil, * A quem a correspondência deve ser enviada
}

sente em sua composição. O licopeno é o carotenóide mais abundante no tomate, sendo o grande responsável por sua coloração vermelha. Ele apresenta um poder antioxidante muito elevado e é associado à prevenção contra alguns tipos de câncer, entre eles o de próstata, do trato digestivo e do pulmão [22].

Recentemente, no Brasil, o consumo de tomate seco vem crescendo de maneira considerável. Atualmente, este produto está presente no cardápio de vários restaurantes e em muitas lojas de comidas finas. Geralmente, o tomate seco é produzido por pequenos e microempresários, que utilizam métodos de produção artesanais, mantidos em segredo para evitar a concorrência. No entanto, quando se trata de médios e grandes volumes de produção, torna-se importante o desenvolvimento de um processo que, além de resultar num produto de alta qualidade, seja viável economicamente e de fácil transposição de escala.

A secagem é um processo que normalmente envolve o uso de temperaturas elevadas, que podem provocar a degradação e a oxidação de alguns nutrientes. Neste contexto, a desidratação osmótica se destaca como um pré-tratamento eficiente, uma vez que promove um melhora na qualidade do produto final, acarretando uma redução da perda de 
nutrientes como vitaminas, minerais e mesmo de licopeno [22]. Além disso, apresenta vantagens como a economia de energia (redução do tempo de secagem) e o fato de o produto ser processado em fase líquida, fornecendo bons coeficientes de transferência de calor e de massa [19].

A desidratação osmótica é um método de remoção parcial de água dos alimentos. Baseia-se na imersão dos alimentos em soluções hipertônicas de um ou mais solutos, originando dois fluxos simultâneos e opostos: uma saída de água do produto para a solução e uma migração de solutos da solução para o produto. A saída de sólidos naturais do alimento é quantitativamente desprezível, embora possa ser importante no que diz respeito às características sensoriais e nutricionais. De acordo com RASTOGI \& RAGHAVARAO [20], a força motriz responsável pela saída de água é a diferença de pressão entre o produto e a solução osmótica, enquanto, no caso da penetração dos solutos, a força motriz é a diferença de concentração entre eles.

Em alimentos, o processo osmótico envolve a utilização de soluções com um ou mais solutos, que, normalmente, apresentam viscosidade elevada. A viscosidade é uma propriedade física muito importante, pois exerce grande influência sobre o nível de agitação necessário ao processo, que representa um importante aspecto econômico e merece, portanto, ser estudado. Além disso, a viscosidade afeta também a taxa de transferência de massa do processo. Quando o meio osmótico é altamente viscoso, a transferência de massa pode ser dificultada e, desse modo, a consideração de que a resistência externa à transferência de massa é desprezível (presente na maioria dos modelos que descrevem a desidratação osmótica) nem sempre é válida [7]. Normalmente, costuma-se conduzir o processo de desidratação osmótica sob agitação, visando-se minimizar os efeitos desta resistência externa.

Os solutos mais utilizados no processo de desidratação osmótica de frutas e vegetais são açúcares e sais, principalmente sacarose e cloreto de sódio [10, 13, 23]. A sacarose é considerada o melhor agente desidratante, principalmente quando a desidratação é utilizada como pré-tratamento para a secagem. Sua presença na superfície do material representa um obstáculo ao contato com o oxigênio, resultando em uma redução do escurecimento enzimático. O cloreto de sódio também é considerado um ótimo agente desidratante, por apresentar uma alta capacidade de redução da atividade de água, fazendo com que a força motriz de saída de água do produto seja maior. No entanto, os produtos tratados com este soluto, normalmente, apresentam elevado ganho de sólidos, decorrente de seu baixo peso molecular, o que torna seu uso limitado a alguns frutos e vegetais, devido ao sabor salgado que ele confere. Vários autores têm apontado o uso de misturas de solutos como um método vantajoso, que permite a obtenção de altos níveis de perda de água, com uma pequena incorporação de solutos [8, 15, 17]. Além disso, de acordo com BOHUON et al. [5], a desidratação

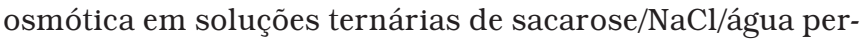
mite o uso de concentrações de solutos maiores, sem que se atinja o limite de saturação das soluções.
Desta forma, o objetivo deste trabalho foi estudar a influência da agitação, da temperatura e da composição da solução sobre a perda de água e a incorporação de sacarose e cloreto de sódio, bem como sobre a atividade de água do produto final, na desidratação osmótica de metades de tomate em soluções ternárias. A atividade de água das amostras foi também relacionada à atividade de água das soluções osmóticas, com a finalidade de se verificar a proximidade do equilíbrio apresentada por cada amostra.

\section{2 - MATERIAL E MÉTODOS}

\section{1 - Material}

\subsection{1 - Matéria-prima}

Como matéria-prima, foram utilizados tomates maduros da variedade Deborah (composição apresentada na Tabela 1), adquiridos no mercado local (CEASA - Campinas, $\mathrm{SP})$ e selecionados com base no tamanho $(58,4 \pm 3,1 \mathrm{~mm}$ de comprimento, 58,1 $\pm 2,2 \mathrm{~mm}$ de diâmetro e peso de $131,19 \pm 12,15 \mathrm{~g}$ ), intensidade da cor e firmeza, de forma a se obterem amostras relativamente homogêneas.

Os tomates foram lavados em água corrente e sanitizados em uma solução de desinfetante clorado para frutas e verduras - Sumaveg (Diversey Lever) -, numa concentração de $100 \mathrm{ppm}$, durante $10 \mathrm{~min}$. Em seguida, foram cortados em metades, tiveram as sementes removidas $\mathrm{e}$ foram pesados.

TABELA 1 - Composição do tomate (variedade Deborah).

\begin{tabular}{lcc}
\hline \multicolumn{1}{c}{ Componente } & Valor médio (\%) & Método de análise \\
\hline Água & $94,49 \pm 0,44$ & Gravimetria \\
Proteínas & $0,87 \pm 0,06$ & {$[18]$} \\
Lipídeos & $0,12 \pm 0,02$ & {$[4]$} \\
Açúcares redutores & $3,92 \pm 0,64$ & {$[18]$} \\
Açúcares totais & $3,92 \pm 0,64$ & {$[18]$} \\
Cinzas & $0,42 \pm 0,01$ & {$[2]$} \\
Acidez titulável (\% ácido cítrico) & $0,28 \pm 0,01$ & {$[18]$} \\
\hline
\end{tabular}

\subsection{2 - Soluções osmóticas}

As soluções desidratantes foram preparadas com água destilada, sacarose e cloreto de sódio comerciais, numa concentração total de $65 \%(\mathrm{p} / \mathrm{p})$, sendo que a quantidade de cada soluto variou de $0 \%$ a $10 \%$ e de $55 \%$ a $65 \%$ para o sal e o açúcar, respectivamente. A relação amostra: solução utilizada foi de 1:15 (p/p), a fim de se evitar a diluição da solução durante o processo.

\section{2 - Métodos}

\subsection{1 - Desidratação osmótica}

O processo de desidratação osmótica foi realizado em um tanque encamisado de aço inoxidável, de $220 \mathrm{~mm}$ de altura 
e 235 mm de diâmetro interno, conforme mostra a Figura 1. O tanque foi conectado a um banho termostático (PolyScience 9500 Series, Niles, EUA), com a finalidade de manter a temperatura da solução osmótica constante. A agitação da solução foi promovida por um agitador mecânico, utilizandose uma turbina de $95 \mathrm{~mm}$ de diâmetro, sendo a velocidade de rotação da turbina controlada por um tacômetro digital (Tecnal, modelo TE-039/1, Piracicaba, Brasil).

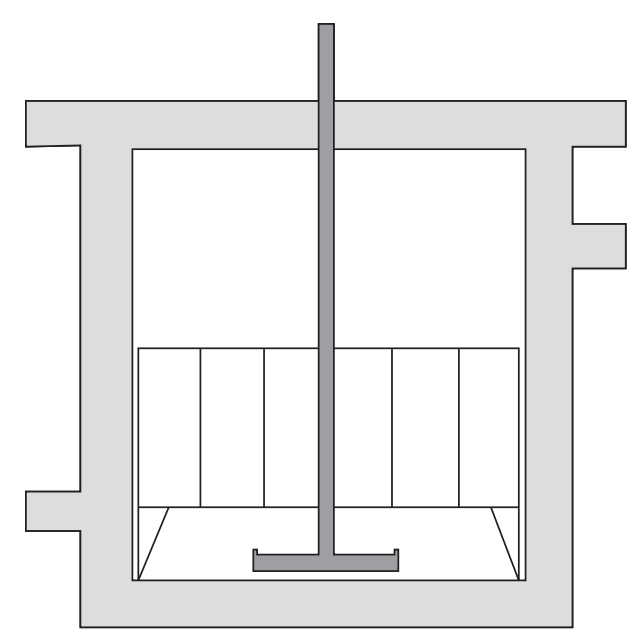

FIGURA 1 - Tanque usado para os ensaios de desidratação osmótica.

As metades de tomates foram colocadas em uma cesta de aço inox e imersas na solução previamente aquecida à temperatura de processo. Após $3 \mathrm{~h}$ de processo (período em que, de acordo com BARONI [3], ocorrem as maiores taxas de transferência de massa no tomate), as amostras foram retiradas, enxaguadas com água destilada, colocadas em papel absorvente para retirar o excesso de solução e pesadas, para serem, então, analisadas quanto à perda de peso $(P P)$, perda de água ( $P A)$, ganho de sal (GSal) e ganho de sacarose (GSac), de acordo com as Equações de (1) a (4):

$$
\begin{aligned}
& P P(\%)=\frac{M_{o}-M_{t}}{M_{o}} * 100 \\
& P A(\%)=\frac{U_{o}{ }^{*} M_{o}-U_{t}{ }^{*} M_{t}}{M_{o}} * 100 \\
& \operatorname{GSal}(\%)=\frac{S a l_{t}-S a I_{o}}{M_{o}} * 100 \\
& \operatorname{GSac}(\%)=P A(\%)-P P(\%)-\text { GSal }(\%)
\end{aligned}
$$

Onde:

$$
\begin{aligned}
& M=\text { massa da amostra (g); } \\
& U \text { = teor de umidade da amostra, em base úmida (\%); } \\
& S a l=\text { conteúdo de cloreto de sódio na amostra (g); e }
\end{aligned}
$$

Os índices o e $t$ representam o processo no início e num determinado tempo $t$, respectivamente.

As amostras, bem como as soluções osmóticas, foram analisadas também em relação à atividade de água, no início e no final do processo.

\subsection{2 - Métodos analíticos}

O teor de umidade das amostras foi determinado pelo método gravimétrico, por secagem em estufa a vácuo, a $60{ }^{\circ} \mathrm{C}$, durante $48 \mathrm{~h}$. O conteúdo de $\mathrm{NaCl}$ foi determinado de acordo com o Método de Mohr [18], baseado na titulação com nitrato de prata, utilizando-se cromato de potássio como indicador. A atividade de água das amostras e das soluções desidratantes foi medida em um higrômetro digital Aqualab, modelo CX-2T (Decagon, Pullman, EUA).

Todas as análises foram realizadas pelo menos em triplicata.

\subsection{3 - Planejamento experimental}

A metodologia de superfície de resposta foi utilizada para avaliar a influência das variáveis de processo sobre a desidratação osmótica das metades de tomate. Os ensaios experimentais foram realizados de acordo com um planejamento fatorial $2^{3}$ completo, com 8 pontos fatoriais (níveis \pm 1 ), 3 pontos centrais (nível 0) e 6 pontos axiais $( \pm \alpha)$, totalizando 17 ensaios. Este planejamento teve como objetivo avaliar o efeito da temperatura, da composição da solução e do nível de agitação (variáveis independentes) sobre as respostas: perda de água (PA), ganho de sal (GSal), ganho de sacarose (GSac), atividade de água $\left(a_{w}\right)$ e relação $a_{\mathrm{w}}$ solução $/ a_{w}$ amostra $\left(a_{w s} / a_{w a}\right.$ ), ao final do processo. Os dados obtidos foram ajustados ao seguinte polinômio:

$Y=\beta_{0}+\beta_{1} T+\beta_{2} C+\beta_{3} A+\beta_{11} T^{2}+$

$\beta_{22} C^{2}+\beta_{33} A^{2}+\beta_{12} T C+\beta_{13} T A+\beta_{23} C A$

Onde $\beta_{n}$ são os coeficientes de regressão, $Y$ é a resposta em questão (PA, GSal, GSac, $a_{w}$ ou $\left.a_{w s} / a_{w a}\right)$ e $T, C$ e $A$ são as variáveis independentes codificadas (temperatura, composição da solução e agitação, respectivamente). As Tabelas 2 e 3 apresentam os valores das variáveis independentes codificadas e os ensaios realizados para o planejamento experimental, respectivamente.

TABELA 2 - Variáveis independentes codificadas.

\begin{tabular}{lccccc}
\hline \multicolumn{1}{c}{ Variáveis } & $-\alpha$ & $-\mathbf{1}$ & $\mathbf{0}$ & $\mathbf{+ 1}$ & $\mathbf{+ \alpha}$ \\
\hline Temperatura $\left({ }^{\circ} \mathrm{C}\right)$ & 20 & 24 & 30 & 36 & 40 \\
$\begin{array}{l}\text { Composição da solução } \\
\text { (\%sal/\%sacarose) }\end{array}$ & $0 / 65$ & $2 / 63$ & $5 / 60$ & $8 / 57$ & $10 / 55$ \\
Agitação (rpm) & 0 & 200 & 500 & 800 & 1000 \\
\hline
\end{tabular}

A análise de variância (ANOVA), a determinação dos coeficientes de regressão e a geração das superfícies de resposta foram realizadas pelo software Statistica 5.0. 
TABELA 3 - Planejamento experimental da desidratação osmótica (níveis codificados).

\begin{tabular}{|c|c|c|c|c|c|c|c|c|}
\hline \multicolumn{4}{|c|}{ Variáveis independentes } & \multicolumn{5}{|c|}{ Variáveis dependentes (Respostas) } \\
\hline Ensaio & $T$ & C & $A$ & $\begin{array}{l}P A \\
(\%)\end{array}$ & $\begin{array}{c}\text { GSal } \\
(\%)\end{array}$ & $\begin{array}{c}\text { GSac } \\
(\%)\end{array}$ & $a_{w}$ & $a_{w s} / a_{w a}$ \\
\hline 01 & -1 & -1 & -1 & 15,41 & 0,34 & 2,02 & 0,983 & 0,855 \\
\hline 02 & +1 & -1 & -1 & 25,90 & 0,38 & 2,33 & 0,981 & 0,856 \\
\hline 03 & -1 & +1 & -1 & 21,25 & 0,93 & 2,59 & 0,979 & 0,781 \\
\hline 04 & +1 & +1 & -1 & 31,76 & 1,48 & 3,63 & 0,969 & 0,789 \\
\hline 05 & -1 & -1 & +1 & 17,83 & 0,40 & 1,31 & 0,981 & 0,856 \\
\hline 06 & +1 & -1 & +1 & 33,66 & 0,43 & 3,52 & 0,980 & 0,857 \\
\hline 07 & -1 & +1 & +1 & 25,85 & 1,20 & 2,40 & 0,975 & 0,785 \\
\hline 08 & +1 & +1 & +1 & 34,52 & 1,34 & 3,39 & 0,969 & 0,789 \\
\hline 09 & $-1,68$ & 0 & 0 & 18,45 & 0,66 & 2,12 & 0,981 & 0,819 \\
\hline 10 & $+1,68$ & 0 & 0 & 34,30 & 0,87 & 3,70 & 0,978 & 0,821 \\
\hline 11 & 0 & $-1,68$ & 0 & 14,86 & 0,00 & 2,28 & 0,984 & 0,879 \\
\hline 12 & 0 & $+1,68$ & 0 & 29,97 & 1,64 & 2,12 & 0,972 & 0,756 \\
\hline 13 & 0 & 0 & $-1,68$ & 22,47 & 0,78 & 2,00 & 0,980 & 0,819 \\
\hline 14 & 0 & 0 & $+1,68$ & 30,28 & 0,77 & 3,25 & 0,975 & 0,824 \\
\hline 15 & 0 & 0 & 0 & 27,06 & 0,86 & 2,89 & 0,977 & 0,822 \\
\hline 16 & 0 & 0 & 0 & 25,50 & 0,86 & 3,46 & 0,978 & 0,821 \\
\hline 17 & 0 & 0 & 0 & 25,25 & 0,90 & 2,83 & 0,978 & 0,821 \\
\hline
\end{tabular}

\section{3 - RESULTADOS E DISCUSSÃO}

Os valores dos coeficientes de regressão obtidos para as respostas estudadas são apresentados na Tabela 4. Eliminando-se os fatores não-significativos, verificou-se a significância da regressão em relação a $95 \%$ de confiança ( $\mathrm{p} \leq 0,05$ ), pelo teste $\mathrm{F}$, na análise de variância (ANOVA). A Tabela 5 apresenta os valores calculados e tabelados de $\mathrm{F}$.

De acordo com a Tabela 5, os modelos obtidos para perda de água, ganho de sal, $a_{w}$ e $a_{w s} / a_{w a}$ apresentaram regressão significativa ( $\mathrm{F}$ calculado superior ao $\mathrm{F}$ tabelado) ao nível de $95 \%$ de confiança. Os coeficientes de determinação $\left(R^{2}\right)$ obtidos para estas respostas foram superiores a 0,92 , indicando um bom ajuste dos dados a estes modelos e permitindo a geração das superfícies de resposta.

O modelo obtido para o ganho de sacarose, no entanto, apresentou um coeficiente de determinação baixo $(0,6937)$, indicando que a porcentagem de variação explicada pela regressão é de 69,37\% e, portanto, não há um bom ajuste dos

TABELA 4 - Coeficientes de regressão obtidos para PA, GSal, GSac, $\mathrm{a}_{\mathrm{w}}$ e $a_{\mathrm{ws}} / a_{\mathrm{wa}}$.

\begin{tabular}{|c|c|c|c|c|c|}
\hline Coeficientes & $P A$ & GSal & GSac & $a_{w}$ & $a_{w s} / a_{w a}$ \\
\hline$\beta_{0}$ & 25,883 & 0,871 & 3,053 & 0,978 & 0,821 \\
\hline$\beta_{1}$ & 5,286 & 0,082 & 0,528 & $-1,76 \times 10^{-3}$ & $1,45 \times 10^{-3}$ \\
\hline$\beta_{2}$ & 3,371 & 0,451 & 0,188 & $-3,89 \times 10^{-3}$ & $-0,036$ \\
\hline$\beta_{3}$ & 2,247 & NS & NS & $-1,13 \times 10^{-3}$ & $9,44 \times 10^{-4}$ \\
\hline$\beta_{11}$ & NS & $-0,031$ & NS & NS & NS \\
\hline$\beta_{22}$ & $-1,075$ & NS & $-0,282$ & NS & $-9,59 \times 10^{-4}$ \\
\hline$\beta_{33}$ & NS & NS & NS & NS & NS \\
\hline$\beta_{12}$ & $-0,893$ & 0,078 & NS & $-1,62 \times 10^{-3}$ & $1,29 \times 10^{-3}$ \\
\hline$\beta_{13}$ & NS & $-0,053$ & 0,231 & NS & NS \\
\hline$\beta_{23}$ & NS & NS & NS & NS & NS \\
\hline
\end{tabular}

NS: Não-significativos $(p>0,05)$.

TABELA 5 - Análise de variância para PA, GSal, GSac, $a_{\mathrm{w}}$ e $a_{\mathrm{ws}} / a_{\mathrm{wa}}$, na desidratação osmótica de metades de tomate.

\begin{tabular}{|c|c|c|c|c|c|}
\hline \multirow{2}{*}{$\begin{array}{c}\text { Fontes de } \\
\text { variação }\end{array}$} & $S Q$ & GL & $M Q$ & $F_{\text {calculado }}$ & $F_{\text {tabelado }}{ }^{*}$ \\
\hline & \multicolumn{5}{|c|}{$P A$} \\
\hline Regressão & 631,02 & 5 & 126,20 & 46,23 & 3,20 \\
\hline Resíduo & 30,04 & 11 & 2,73 & - & - \\
\hline Total & \multicolumn{5}{|c|}{ GSal } \\
\hline Regressão & 2,944 & 5 & 0,5888 & 113,23 & 3,20 \\
\hline Total & \multicolumn{5}{|c|}{ GSac } \\
\hline Regressão & 5,57 & 4 & 1,39 & 6,95 & 3,26 \\
\hline Resíduo & 2,42 & 12 & 0,20 & - & - \\
\hline \multirow[t]{2}{*}{ Total } & 7,99 & 16 & - & - & $R^{2}=0,6937$ \\
\hline & \multicolumn{5}{|c|}{$a_{w}$} \\
\hline Regressão & $2,88 \times 10^{-4}$ & 4 & $7,20 \times 10^{-5}$ & 36,18 & 3,26 \\
\hline Total & \multicolumn{5}{|c|}{$a_{w s} / a_{w a}$} \\
\hline Regressão & $1,74 \times 10^{-2}$ & 5 & $3,48 \times 10^{-3}$ & 1303,37 & 3,20 \\
\hline Resíduo & $2,94 \times 10^{-5}$ & 11 & $2,67 \times 10^{-6}$ & - & \\
\hline Total & $1,74 \times 10^{-2}$ & 16 & - & - & $R^{2}=0,9983$ \\
\hline
\end{tabular}

$\mathrm{SQ}=$ soma quadrática; $\mathrm{GL}=$ graus de liberdade; $\mathrm{MQ}=$ média quadrática; $\mathrm{e}$ * Valores tabelados de $\mathrm{F}$ a $\mathrm{p} \leq 0,05$ 
dados experimentais ao modelo, o que pode ser confirmado pelo valor de F calculado. De acordo com BOX \& WETZ [6], um modelo pode ser considerado preditivo quando apresenta um valor de $\mathrm{F}$ calculado superior a 3 vezes o valor de $\mathrm{F}$ tabelado, o que não ocorre neste caso. Além disso, conforme mostra a Tabela 5, no caso de GSac, a soma quadrática dos resíduos foi alta, representando aproximadamente $30 \%$ do valor total, ao contrário das outras respostas estudadas. Sendo assim, os coeficientes obtidos indicam apenas uma tendência de comportamento dessa resposta em relação às variáveis estudadas, não sendo adequados para ajustar um modelo e gerar superfícies de resposta.

\section{1 - Perda de água}

A Figura 2 mostra as superfícies de resposta para a perda de água, geradas pelo modelo proposto, considerando-se os pontos médios de temperatura, composição da solução e agitação.

A temperatura foi a variável que apresentou maior influência sobre a perda de água ao final de $3 \mathrm{~h}$ de processo, resultando num aumento desta resposta. Este fato se deve ao possível inchaço e plasticização sofridos pela membrana celular durante a desidratação osmótica a temperaturas mais altas, que acarretam um aumento de sua permeabilidade, conforme observado por LAZARIDES et al. [11]. Além disso, o aumento da temperatura acarreta uma redução da viscosidade da solução osmótica, o que faz com que a resistência externa à transferência de massa seja menor.

Em relação à composição da solução, quanto maior o teor de sal utilizado, maior foi a perda de água apresentada pelas amostras. A presença do $\mathrm{NaCl}$ provoca uma redução da atividade de água da solução, aumentando, assim, a força motriz que provoca a saída de água do produto. De acordo com SERENO, MOREIRA \& MARTÍNEZ [21], o sal também pode provocar mudanças estruturais na membrana celular, alterando suas propriedades físicas e levando a um aumento em sua permeabilidade, principalmente a temperaturas mais elevadas. Além disso, segundo LENART \& FLINK [12], a presença de sal inibe a formação de uma barreira de açúcar na superfície do alimento, incorrendo também em maiores taxas de desidratação.

A agitação também mostrou influenciar positivamente a saída de água do produto durante o processo. Isso indica a presença de uma resistência externa à transferência de massa da água na solução osmótica, provavelmente relacionada à sua alta viscosidade, o que sugere que o processo de saída de água do tomate não seja controlado apenas por um mecanismo interno de difusão. Desta maneira, a condição utilizada na solução da Lei de Fick, de que a resistência externa ao transporte de massa pode ser considerada desprezível, neste caso não é verdadeira. Resultados semelhantes foram obtidos por BOHUON et al. [5], trabalhando com um disco de gel. Os autores observaram um aumento da perda de água com o aumento da velocidade de rotação do disco.
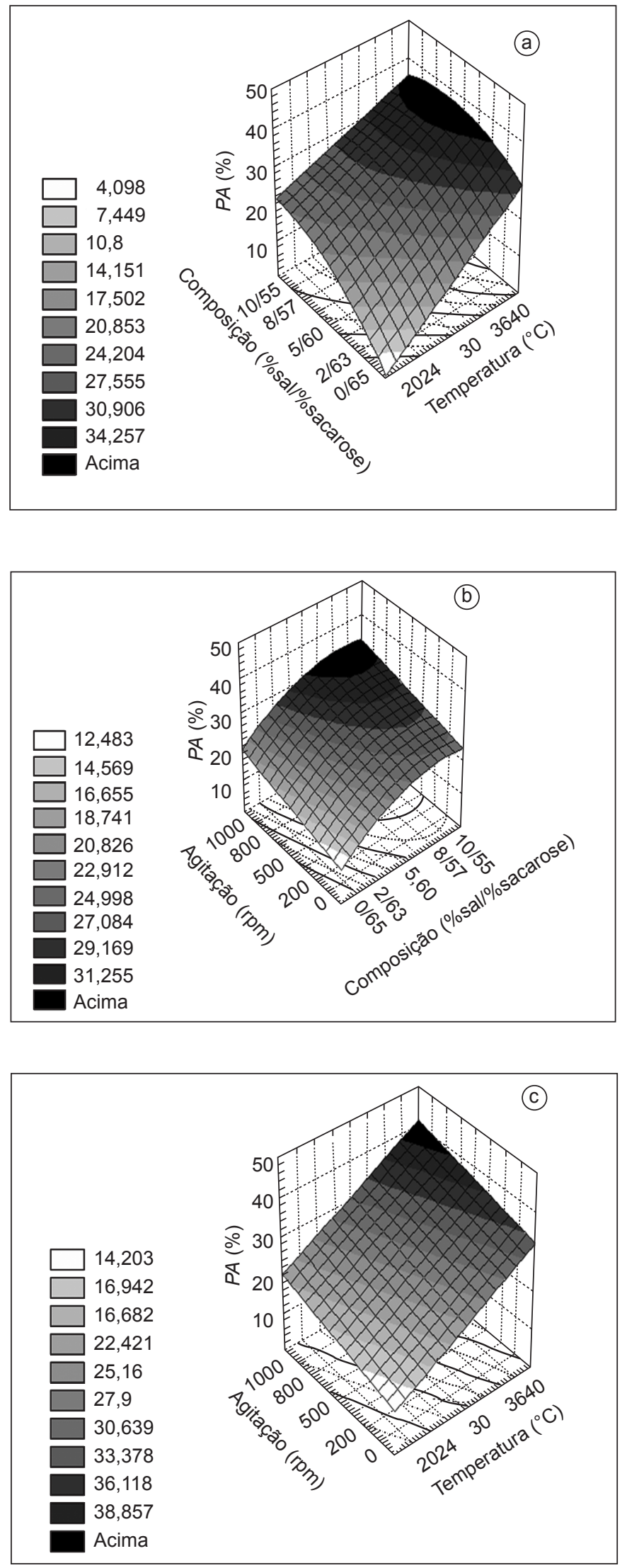

FIGURA 2 - Superfícies de resposta para $P A$ a: a) 500 rpm de agitação; b) solução com $5 \%$ sal $/ 60 \%$ sacarose; e c) $30{ }^{\circ} \mathrm{C}$. 


\section{2 - Ganho de sal}

As superfícies de resposta geradas pelo modelo proposto para o ganho de sal são apresentadas na Figura 3, na qual se observa claramente que a composição da solução foi o fator que exerceu maior influência sobre a incorporação de sal pelo produto. Embora a diferença entre as quantidades de sal utilizadas em cada solução tenha sido pequena, o efeito causado sobre a penetração deste soluto no alimento é considerável, devido, principalmente, ao seu baixo peso molecular, que facilita seu transporte.

O aumento da temperatura também provocou um aumento na transferência de massa do $\mathrm{NaCl}$, fato que, assim como no caso da água, provavelmente está relacionado ao aumento da permeabilidade da membrana celular e à redução da viscosidade da solução osmótica.

A agitação não apresentou influência significativa sobre GSal, assim como foi observado por BOHUON et al. [5], indicando que a transferência de massa do cloreto de sódio no tomate, provavelmente, é governada por um mecanismo predominantemente difusivo e que a única resistência apresentada ao transporte deste soluto é a resistência encontrada no interior do alimento. Portanto, neste caso, a resistência externa à transferência de massa pode ser considerada desprezível.

\section{3 - Ganho de sacarose}

De acordo com os coeficientes apresentados na Tabela 4, a temperatura linear foi o fator que mais influenciou a incorporação de sacarose, novamente devido ao aumento da permeabilidade da membrana e da diminuição da viscosidade da solução osmótica.

A composição da solução também influenciou positivamente o transporte da sacarose durante o processo. O aumento no teor de sal presente na solução parece ter provocado um aumento na incorporação de sacarose. Este fato pode estar relacionado ao aumento da permeabilidade da membrana celular, decorrente das alterações físicas provocadas pelo sal [21], que pode acarretar uma maior penetração de sacarose no produto. Além disso, à medida que o teor de sal na solução diminui, o teor de açúcar aumenta (uma vez que a concentração total de solutos é sempre a mesma), o que pode provocar a formação de uma barreira sólida de açúcar na superfície do produto, dificultando a penetração da sacarose em seu interior.

A agitação não apresentou influência significativa sobre o ganho de sacarose, quando considerada isoladamente, indicando que a transferência de massa da sacarose, assim como a de sal, é governada, predominantemente, por um mecanismo de transporte interno e a resistência externa ao transporte de massa, neste caso, também pode ser considerada desprezível. Resultados semelhantes foram obtidos por Moreira e Sereno [16], trabalhando com desidratação osmótica de cilindros de maçã.
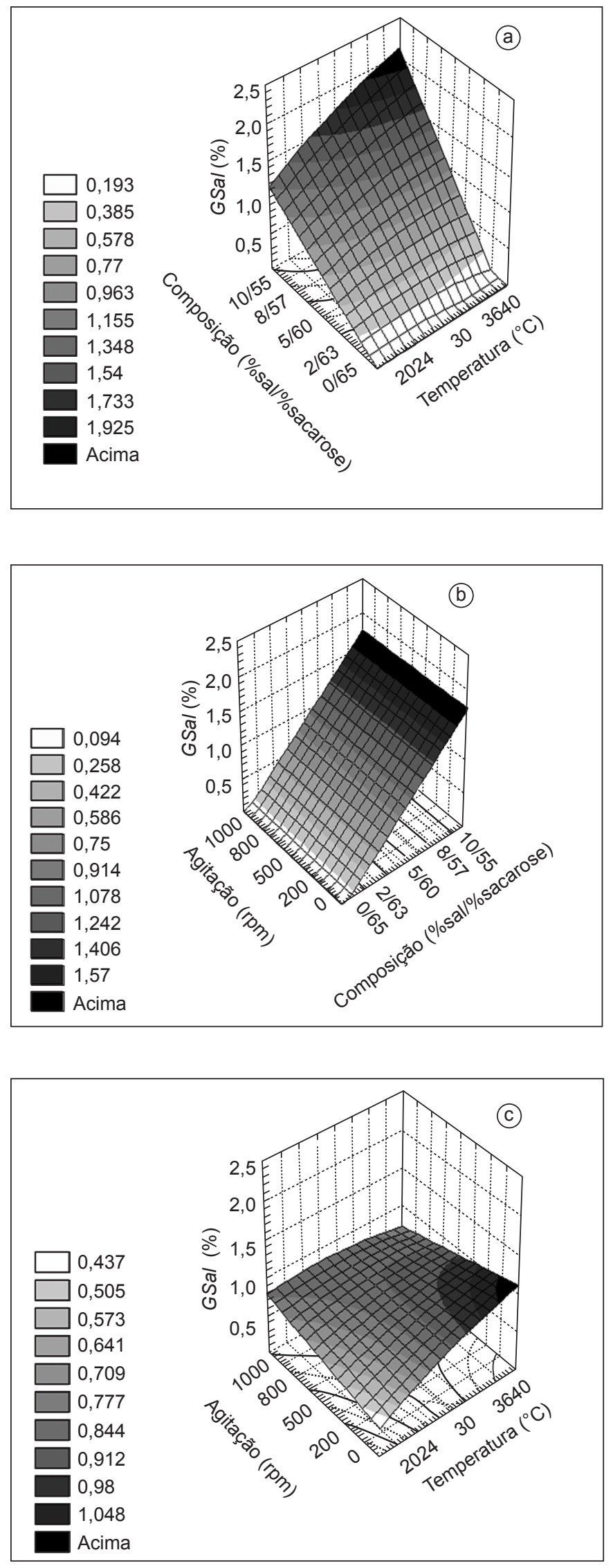

FIGURA 3 - Superfícies de resposta para GSal a: a) $500 \mathrm{rpm}$ de agitação; b) solução com $5 \% \mathrm{sal} / 60 \%$ sacarose; e c) $30{ }^{\circ} \mathrm{C}$. 


\section{4 - Atividade de água}

As superfícies de resposta geradas pelo modelo proposto para a atividade de água são apresentadas na Figura 4.

Entre as variáveis estudadas, a composição da solução foi a que apresentou maior influência sobre a atividade de água final do produto. O aumento do teor de sal na solução, além de promover uma maior saída de água, também ocasionou uma maior incorporação deste soluto pelo produto, como discutido anteriormente. A perda de água, aliada à alta capacidade apresentada pelo sal de reduzir a $a_{w}$, faz com que esta resposta seja menor, à medida que a concentração de $\mathrm{NaCl}$ na solução aumenta. A influência do cloreto de sódio sobre a atividade de água do tomate desidratado osmoticamente pode ser explicada pelo fato deste soluto ser uma substância eletrolítica, que, portanto, se dissocia em íons, que interagem com as moléculas de água, provocando uma redução da atividade de água.

A temperatura e a interação temperatura x composição também apresentaram forte influência sobre a atividade de água dos produtos, fato que também pode ser relacionado à maior perda de água e incorporação de sal ocasionados em maiores temperaturas.

A influência da agitação foi menor quando comparada aos outros dois fatores, provavelmente porque esta variável exerce influência significativa apenas sobre a saída de água e não sobre a incorporação de $\mathrm{NaCl}$ pelo produto.

\section{5 - Atividade de água da amostra/Atividade de água da solução}

No processo de desidratação osmótica, o equilíbrio é atingido quando a atividade de água do produto se iguala à atividade de água da solução. Levando-se em conta que a solução osmótica apresenta uma atividade de água inferior à das amostras e considerando-se a relação $a_{w}$ solução/ $a_{w}$ amostra $\left(a_{w s} / a_{w a}\right)$ como uma resposta a ser avaliada, os valores desta relação podem representar uma indicação do quão próximo do equilíbrio o processo chegou. Desta forma, quanto maior for o valor desta relação (ou seja, quanto mais próximo de 1), mais próximo o processo de desidratação osmótica chegou do equilíbrio.

A Figura 5 mostra as superfícies de resposta para a relação $a_{w s} / a_{w a}$, geradas pelo modelo proposto, considerando-se os pontos médios de temperatura, composição da solução e agitação.

As Figuras 5(a) e 5(c) tornam evidente a grande influência da composição da solução em relação à proximidade do equilíbrio atingida por cada amostra, em comparação à temperatura e à agitação da solução, que exercem uma influência muito pequena. Guanto maior o teor de sal presente na solução osmótica, menor foi o valor de $\left(a_{w s} / a_{w a}\right)$, ou seja, mais distante do equilíbrio o processo ficou, ao final de $3 \mathrm{~h}$.

MEDINA-VIVANCO [14], em seu trabalho sobre desidratação osmótica de filés de tilápia, observou que quanto
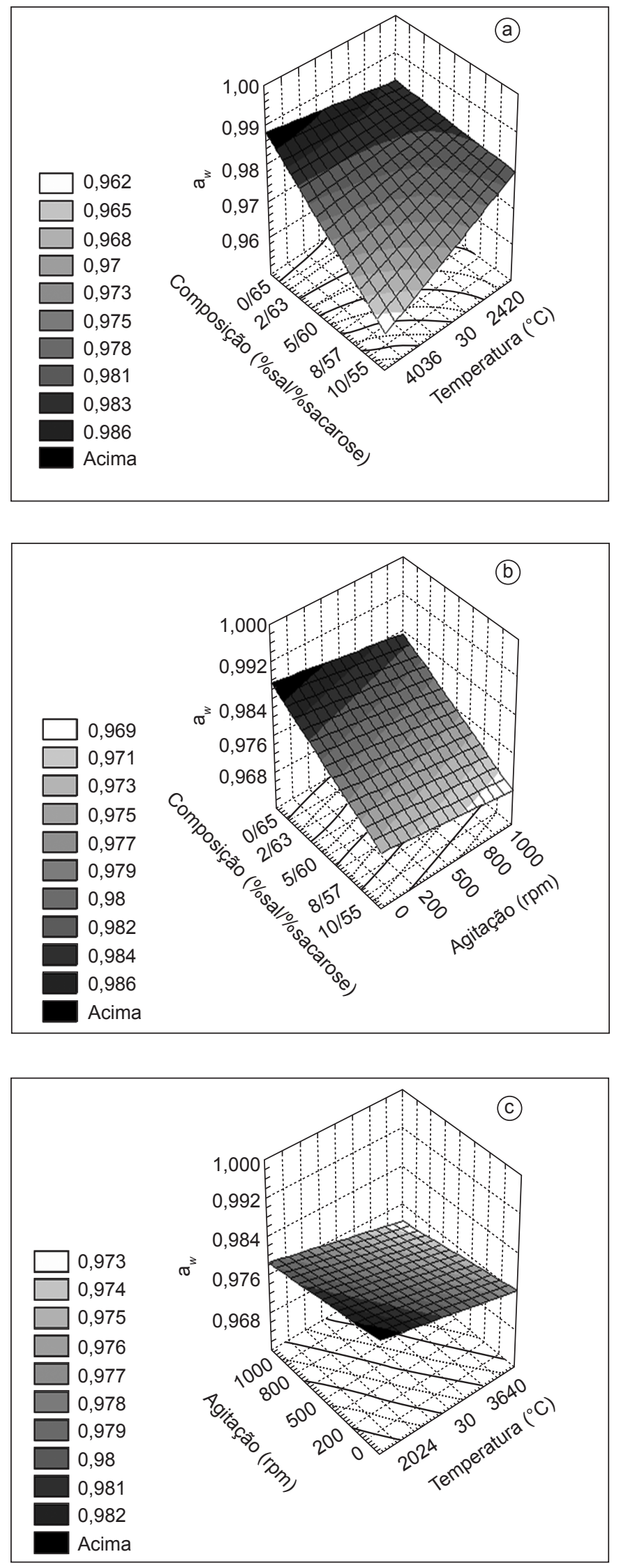

FIGURA 4 - Superfícies de resposta para $\mathrm{a}_{w}$ a: a) 500 rpm de agitação; b) solução com $5 \%$ sal/60\% sacarose; e c) $30{ }^{\circ} \mathrm{C}$. 

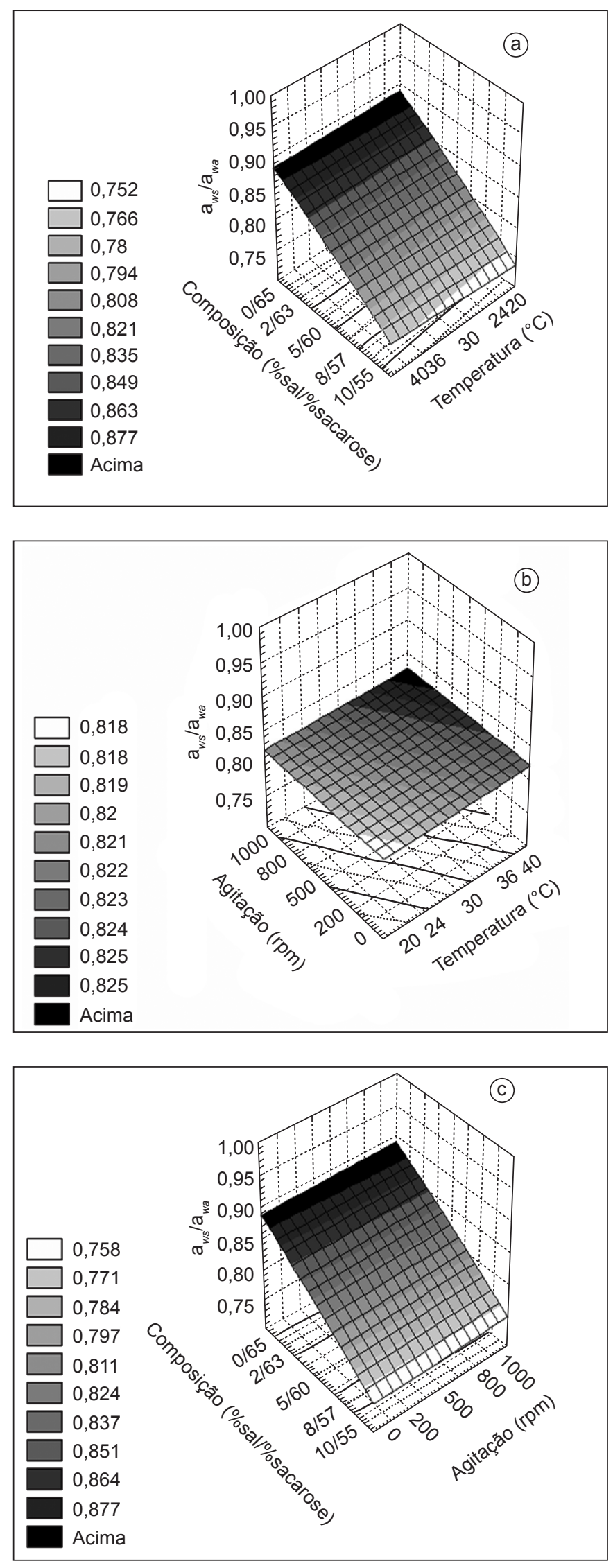

FIGURA 5 - Superfícies de resposta para $\mathrm{a}_{w s} / \mathrm{a}_{w a}$ a: a) $500 \mathrm{rpm}$ de agitação; b) solução com $5 \% \mathrm{sal} / 60 \%$ sacarose; e c) $30{ }^{\circ} \mathrm{C}$. maior o teor de sal presente na solução inicial, mais rápido o sistema atingiu o equilíbrio. Isso pode ser explicado pelo fato de ter sido utilizada uma relação amostra: solução de $1: 4$, que faz com que a solução seja mais rapidamente diluída (com a saída de água do produto, que aumenta com o aumento do teor de $\mathrm{NaCl}$ ). Conseqüentemente, sua atividade de água apresenta um aumento mais rápido, se aproximando mais da atividade de água do produto. Já neste trabalho, a relação amostra: solução utilizada foi de 1:15, justamente para evitar a diluição da solução durante o processo. Sendo assim, mesmo com a maior perda de água apresentada pelas amostras tratadas em solução com maior teor de sal, esta perda não foi suficiente para aumentar consideravelmente a atividade de água da solução. Isso explica o fato de as amostras processadas em solução binária de sacarose se apresentarem "mais próximas" do equilíbrio após $3 \mathrm{~h}$ de processo. No entanto, não é possível concluir qual das amostras atinge o equilíbrio mais rapidamente, uma vez que seriam necessários dados de cinética de atividade de água da solução e do produto em tempos muito superiores aos estudados.

\section{4 - CONCLUSÕES}

Todas as respostas analisadas foram influenciadas positivamente pela temperatura e pela composição da solução osmótica. A agitação apresentou efeito positivo sobre a perda de água, mas não sobre a incorporação de sal e de sacarose, o que sugere que o processo de transferência de massa da água não é controlado apenas internamente e que a condição de resistência externa à transferência de massa desprezível, adotada na maioria das soluções da Segunda Lei de Fick, não pode ser considerada neste caso. Já o transporte dos solutos, ao contrário, parece ser governado predominantemente por um mecanismo interno, uma vez que não sofre influência do nível de agitação do processo.

Em relação à proximidade do equilíbrio atingida pelas amostras, observou-se que os ensaios realizados em soluções com maior teor de cloreto de sódio apresentaram-se mais distantes do equilíbrio após $3 \mathrm{~h}$ de desidratação osmótica.

\section{5 - REFERÊNCIAS BIBLIOGRÁFICAS}

[1] AGRIANUAL 2003. Anuário da Agricultura Brasileira. São Paulo: FNP - Consultoria e Comércio, 2002.

[2] A.O.A.C. (Association of Official Analytical Chemists) - Official Methods of Analysis of the Association of Official Analytical Chemists, $16^{\text {th }}$ edition. Arlington: A.O.A.C., 1995.

[3] BARONI, A. F. Propriedades mecânicas, termodinâmicas e de estado de tomate submetido à desidratação osmótica e secagem. Campinas, 2004, 226 p. Tese (Doutor em Engenharia de Alimentos) - Faculdade de Engenharia de Alimentos, Universidade Estadual de Campinas (UNICAMP).

[4] BLIGH, E. G.; DYER, W. J. A rapid method of total lipid extraction and purification. Canadian Journal of Biochemistry and Physiology, v. 37, n. 8, p. 911-917, 1959. 
[5] BOHUON, P.; COLlignAN, A.; RIOS, G. M.; RAOULTWACK, A. L. Soaking process in ternary liquids: experimental study of mass transport under natural and forced convection. Journal of Food Engineering, v. 37, n. 4, p .451-469, 1998.

[6] BOX, G. E. P.; WETZ, J. Criteria for judging adequacy of estimation by an approximate response function. University of Wiscosin Technical Report, n. 9, 1973.

[7] CHENLO, F.; MOREIRA, G.; PEREIRA, G.; AMPUDIA, A. Viscosities of aqueous solutions of sucrose and sodium chloride of interest in osmotic dehydration processes. Journal of Food Engineering, v. 54, n. 4, p. 347-352, 2002.

[8] COLlignAN, A.; RAOULT-WACK, A. L. Dewatering and salting of cod by immersion in concentrated sugar/salt solutions. Lebensmittel-Wissenschaft und Technologie, v. 27, n. 3, p. 259-264, 1994.

[9] EMBRAPA - HORTALIÇAS. Hortaliças em números - Dados socioeconômicos. Disponível em: <www.cnph. embrapa.br > . Acesso em: 20 de novembro de 2005.

[10] GIRALDO, G.; TALENS, P.; FITO, P.; CHIRALT, A. Influence of sucrose solution concentration on kinetics and yield during osmotic dehydration of mango. Journal of Food Engineering, v. 58, n. 1, p. 33-43, 2003.

[11] LAZARIDES, H. N.; KATSANIDIS, E.; NICKOLAIDIS, A. Mass transfer kinetics during osmotic preconcentration aiming at minimal solid uptake. Journal of Food Engeneering, v. 25, n. 2, p. 151-166, 1995.

[12] LENART, A.; FLINK, J. N. Osmotic concentration of potatoes: Criteria for the end point of the osmotic effect. Journal of Food Technology, v. 19, n. 1, p. 65-89, 1984.

[13] MAYOR, L.; MOREIRA, R.; CHENLO, F.; SERENO, A. M. Kinetics of osmotic dehydration of pumpkim with sodium chloride solutions. Journal of Food Engineering, v. 74, n. 2, p. 253-262, 2005.

[14] MEDINA-VIVANCO, M. L. Desidratação osmótica em soluções ternárias, secagem e transições térmicas de filé de tilápia (Oreochromis niloticus). Campinas, 2003, 211 p. Tese (Doutor em Engenharia de Alimentos) - Faculdade de Engenharia de Alimentos, Universidade Estadual de Campinas (UNICAMP).

[ 15]MEDINA-VIVANCO, M.; SOBRAL, P. J. A.; HUBINGER, M. D. Osmotic dehydration of tilapia fillets in limited volume of ternary solutions. Chemical Engineering Journal, v. 86, n. 1-2, p. 199-205, 2002.

[16] MOREIRA, R.; SERENO, A. M. Evaluation of mass transfer coefficients and volumetric shrinkage during osmotic dehydration of apple using sucrose solutions in static and non-static conditions. Journal of Food Engineering, v. 57, n. 1, p. 25-31, 2003.

[17] QI, H.; LE MAGUER, M.; SHARMA, S. K. Design and selection of processing conditions of a pilot scale contactor for continuous osmotic dehydration of carrots. Journal of Food Process Engineering, v. 21, n. 1, p. 75-88, 1998.

[18] RANGANNA, S. Manual of analysis of fruit and vegetable products, New Delhi, India: Mc Graw-Hill Publishing Company Limited, 1977.

[19] RAOULT-WACK, A. L. Recent advances in the osmotic dehydration of foods. Trends in Food Science \& Technology, v. 5, n. 8, p. 255-260, 1994.

[20] RASTOGI, N. K.; RAGHAVARAO, K. S. M. S. Mass transfer during osmotic dehydration of pineapple: considering Fickian diffusion in cubical configuration. Lebensmittel-Wissenschaft und Technologie, v. 37 , n. 1, p. 43-47, 2004.

[21] SERENO, A. M.; MOREIRA, R.; MARTÍnEZ, E. Mass transfer coefficients during osmotic dehydration of apple in single and combined aqueous solutions of sugar and salt. Journal of Food Engineering, v. 47, n. 1, p. 43-49, 2001.

[22] SHI, J.; LE MAGUER, M.; KAKUDA, Y.; LIPTAY, A.; NIEKAMP, F. Lycopene degradation and isomerization in tomato dehydration. Food Research International, v. 32, n. 1, p. 15-21, 1999.

[23] TELIS, V. R. N.; MURARI, R. C. B. D. L.; YAMASHITA, F. Diffusion coefficients during osmotic dehydration of tomatoes in ternary solutions. Journal of Food Engineering, v. 61, n. 2, p. 253-259, 2004.

\section{6 - AGRADECIMENTOS}

Os autores agradecem ao Conselho Nacional de Desenvolvimento Científico e Tecnológico (CNPq), à Coordenação de Aperfeiçoamento de Pessoal de Nível Superior (CAPES) e à Fundação de Amparo à Pesquisa do Estado de São Paulo (FAPESP), pelo apoio financeiro. 
